\title{
Clinical and Epidemiological Study of Intracranial Tumors in Children and Identification of Diagnostic Biomarkers for the Most Common Tumor Subtype and Their Relationship With the Immune Microenvironment Through Bioinformatics Analysis
}

\author{
Guanyi Wang \\ Xijing Hospital https://orcid.org/0000-0003-1202-4651 \\ Yibin Jia \\ Xijing Hospital \\ Yqin Ye \\ Xijing Hospital \\ Enming Kang \\ Xijing Hospital \\ Huijun Chen \\ Xijing Hospital \\ Jiayou Wang \\ Xijing Hospital \\ Xiaosheng He ( $\checkmark$ wgyfmmu@163.com ) \\ Xijing Hospital https://orcid.org/0000-0002-6581-4043
}

\section{Research Article}

Keywords: Brain tumor, Childhood, Pilocytic astrocytoma (PA), biomarker, tumor microenvironment (TME)

Posted Date: February 16th, 2022

DOI: https://doi.org/10.21203/rs.3.rs-1336860/v1

License: () (1) This work is licensed under a Creative Commons Attribution 4.0 International License. Read Full License 


\section{Abstract}

Background: Brain tumors are the second most common pediatric malignancy and have poor prognosis. Understanding the pathogenesis of tumors at the molecular level is essential for clinical treatment. We conducted a retrospective study on the epidemiology of brain tumors in children based on clinical data obtained from a neurosurgical center. After identifying the most prevalent tumor subtype, we identified new potential diagnostic biomarkers through bioinformatics analysis of the public database.

Methods: All children (0-15 years) with brain tumors diagnosed histopathologically between 2010 and 2020 at the Department of Neurosurgery, Xijing Hospital, were reviewed retrospectively for age distribution, sex predilection, native location, tumor location, symptoms, and histological grade, and identified the most common tumor subtypes. Two datasets (GSE44971 and GSE44684) were downloaded from the Gene Expression Omnibus database, whereas the GSE44971 dataset was used to screen the differentially expressed genes between normal and tumor samples. Gene Ontology, Disease Ontology, and Gene Set Enrichment Analysis enrichment analyses were performed to investigate the underlying mechanisms of differentially expressed genes in the tumor. Combined with methylation data in the GSE44684 dataset, we further analyzed the correlation between methylation and gene expression levels. Two algorithms, LASSO and SVM-RFE, were used to select the hub genes of the tumor. The diagnostic value of the hub genes was assessed using the receiver operating characteristic (ROC) curve. Finally, we further evaluated the relationship between the hub gene and the tumor microenvironment and immune gene sets.

Results: Overall, 650 children from 18 provinces in China were included in this study. The male-to-female ratio was 1.41:1, and the number of patients reached a peak in the 10-15-year-old group (41.4\%). The most common symptoms we encountered in our institute were headache and dizziness 250 (28.2\%), and nausea and vomiting 228 (25.7\%). The predominant location is supratentorial, with a supratentorial to infratentorial ratio of 1.74:1. Low-grade tumors (WHO I/II) constituted $60.9 \%$ of all cases and were predominant in every age group. According to basic classification, the most common tumor subtype is pilocytic astrocytoma (PA). A total of 3264 differentially expressed genes were identified in the GSE44971 dataset, which are mainly involved in the process of neural signal transduction, immunity, and some diseases. Correlation analysis indicated that the expression of 45 differentially expressed genes was negatively correlated with promoter DNA methylation. Next, we acquired five hub genes (NCKAP1L, GPR37L1, CSPG4, PPFIA4, and C8orf46) from the 45 differentially expressed genes by intersecting the LASSO and SVM-RFE models. The ROC analysis revealed that the five hub genes had good diagnostic value for patients with PA (AUC > 0.99). Furthermore, the expression of NCKAP1L was negatively correlated with immune, stromal, and estimated scores, and positively correlated with immune gene sets.

Conclusions: This study, based on the data analysis of intracranial tumors in children in a single center over the past 10 years, reflected the clinical and epidemiological characteristics of intracranial tumors in children in Northwest China to a certain extent. PA is considered the most common subtype of intracranial tumors in children. Through bioinformatics analysis, we suggested that NCKAP1L, GPR37L1, CSPG4, PPFIA4, and C8orf46 are potential biomarkers for the diagnosis of PA.

\section{Introduction}

Among childhood cancers, brain tumors follow acute lymphoblastic leukemia as the second most common disease, and accounts for approximately $20 \%$ of childhood cancers(Kaatsch et al. 2001, Steliarova-Foucher et al. 2004, Suresh et al. 2017). Currently, the predominant treatment is surgical resection, supplemented by radiotherapy and chemotherapy(Solomon et al. 2016). Although the survival rate has improved, the side effects have also greatly reduced the quality of patients' lives, and new treatments are vital(Finlay et al. 1996, Matthias et al. 2008).

Recently, mounting evidence has shown that the TME is genetically stable and available to determine abnormal tissue function, while also playing a key role in the development of malignant tumors(Chen et al. 2015, Quail \& Joyce 2013). Immunity plays an important role in TME(Grivennikov et al. 2010), Inspired by such evidence, we may be able to find a novel approach to the treatment of pediatric brain tumors.

Studies have shown that the incidence rate of intracranial tumors in the Asian population is lower than that in other ethnicities. In addition, the incidence of certain types of brain tumors varies between races(Dabiao et al. 2007, Kuratsu et al. 2001, Wong et al. 2005). Consequently, based on the latest WHO classification and diagnostic criteria for nervous system tumors (2016 Edition)(Villa et al. 2018), we analyzed the clinical data of intracranial tumors in children from the Department of Neurosurgery, Xijing Hospital, Air Force Military Medical University in the past decade and summarized the epidemiological characteristics of childhood brain tumors in the northwest of China. We identified the key genes of the most common subtypes of pediatric brain tumors and analyzed the correlation between these 
genes and immune cells. This study aims to provide a theoretical basis for further improving the early diagnosis and treatment outcomes of children with intracranial tumors in China.

\section{Methods}

\subsection{Epidemiological analysis}

Xijing Hospital is the largest hospital in northwest China, as well as the most comprehensive in terms of specializations. From January 2010 to October 2020, 650 children (0-15 years old) with intracranial tumors 18 came from 18 provinces, including Shaanxi, Gansu, Ningxia, and Shanxi, were treated in the Department of Neurosurgery, Xijing Hospital, Air Force Military Medical University. Only patients with a proven histopathological diagnosis were included in the study. All patients were confirmed to have intracranial tumors. We collected the patients' detailed information from the medical records, including age, sex, symptoms, tumor location, pathological examination results, follow-up adjuvant treatment, and other information. According to the most recent WHO classification and diagnostic criteria for tumors of the central nervous system (CNS) (2016 Edition), all 650 cases were classified and graded. Supratentorial tumors include tumors originating from the cerebral hemisphere, third ventricle, lateral ventricle, sellar region, and pineal region, as well as tumors originating from the cerebellum, brainstem, and fourth ventricle. The tumor grade was divided into low-grade and high-grade tumors: WHO grades I and II were classified as low-grade tumors, and grades III and IV were classified as high-grade tumors. The data were processed and scored using descriptive statistics, and categorical data were described in terms of frequencies and percentages.

\subsection{Data collection}

Two datasets, the gene expression profiling dataset (GSE44971) and the gene methylation dataset (GSE44684), were retrieved from the publicly available GEO database (https://www.ncbi.nlm.nih.gov/geo/). The GSE44971 dataset, which was generated on the GPL11154 platform (Affymetrix Human Genome U133 Plus 2.0 Array), included 35 PA tumor samples and 9 normal cerebellum samples after removing 14 non-cerebellar samples. The GSE44684 dataset contained 39 PA tumor samples and 6 normal cerebellum samples (removed the non-cerebellar samples), which were acquired from the GPL13534 platform (Illumina HumanMethylation450 BeadChip).

\subsection{Analysis of differentially expressed genes (DEG)}

To appraised the DEGs between normal cerebellum and PA tumor samples, we applied the 'limma' package for processing the GSE44971 dataset (Ritchie et al. 2015). Genes with $\mid \log _{2} \mathrm{FC}$ (fold change) $\mid \geq 0.5$, and P-value $<0.05$, were considered to be DEGs, which were presented by heatmap and volcano plot.

\subsection{Functional enrichment of DEGs}

We used the R package 'clusterProfiler' to implement the following enrichment analyses: gene ontology (G0) (Anonymous 2006), disease ontology (DO)(Osborne et al. 2009), and gene set enrichment analysis (GSEA)(Subramanian et al. 2005). The GO term enrichment analysis included three categories: biological process (BP), molecular function (MF), and cellular component (CC). For all analyses, statistically significant thresholds were set at $p<0.05$.

\subsection{Methylation data processing}

The R package, 'ChAMP' was used to identify the differential methylation sites between normal and PA samples. $|\Delta \beta|>2$ and P-value less than $\mathbf{0 . 0 5}$, were set as the cutoff criteria. The distribution of the differential methylation sites is shown in the volcano map. For the correlation analysis, we extracted 28 paired PA samples that contained both DNA methylation and gene expression data from the GSE44971 and GSE44684 datasets.

\subsection{LASSO and SVM-RFE Algorithms}

LASSO is an algorithm that can obtain a more refined model by constructing a penalty function (Friedman et al. 2010). SVM-RFE is a feature selection algorithm based on a support vector machine that ranks the features based on the recursive feature deletion sequence (Huang et al. 2014). In this study, the SVM-RFE (e1071 packages) and LASSO regression (glmnet package) algorithms were used to select the characteristic genes from 45 candidate genes. Ultimately, we obtained the intersection of candidate genes screened by the LASSO and SVM-RFE algorithms for follow-up studies.

\subsection{Immune infiltration analysis}

We performed the ESTIMATE algorithm in the GSE44971 dataset using the 'estimate' package in R to generate Immune-, Stromal-, and Estimate-score. PA samples were divided into high expression and low expression groups according to the median value of the expression 
of five hub genes. Next, we further compared the difference of immune, stromal, and estimate scores in the two groups using the Wilcoxon test, with the grouping was based again on the median value of the expression of five hub genes. The ssGSEA is a method used to calculate individual enrichment scores for each sample and gene set(Barbie et al. 2009). In this study, ssGSEA was used to quantify the enrichment levels of 28 immune-related gene sets in each sample of the GSE44971 dataset. The 28 immune-related gene sets were extracted from the study by Bindea et al. (Bindea et al. 2013).

\subsection{Statistical analysis}

All statistical analyses were performed using R software. The AUC of the ROC curve was used to determine the diagnostic value of the five hub genes. Unless otherwise stipulated, $P<0.05$, was considered statistically significant.

\section{Results}

\subsection{Sex and age distribution}

A total of 650 patients were included in our retrospective study, and we subdivided our patients into four age subgroups: less than 1, 1-5 years, 5-10 years, and 10-15 years. There was a slight overall male predominance, with a male to female ratio of 1.41:1, composed of 380 males (58.5\%) and 270 females (41.5\%). Although the sex ratio varied by age subgroup, males were consistently the dominant group. The number of patients increased steadily with age: $4.3 \%$ in the first year, $16.0 \%$ between 2 and 5 years, 38.3\% between 5 and 10 years, and then reaching a peak between 10 and 15 years (41.4\%). The most common age at presentation was between 10 and 15 years, with a mean of 8.8 years (Fig. 1).

\subsection{Symptoms}

As shown in Table 1, among the most common symptoms in our institute were headache and dizziness ( 250 cases, $28.2 \%$ ), nausea and vomiting (228 cases, $25.7 \%$ ), followed by visual disturbances (104 cases, $11.7 \%$ ), and cerebellar signs ( 80 cases, $9.0 \%$ ). Physical examination or routine examination after head trauma revealed 81 cases of intracranial tumors with no symptoms.

Table 1

Symptoms of children with intracranial tumors

\begin{tabular}{|ll|}
\hline Symptom & $\mathrm{n}(\%)$ \\
\hline Asymptomatic & $81(9.1)$ \\
\hline Epilepsy & $56(6.3)$ \\
\hline Headache and dizziness & $250(28.2)$ \\
\hline Nausea and vomiting & $228(25.7)$ \\
\hline Visual disturbances & $104(11.7)$ \\
\hline Cerebellar signs & $80(9.0)$ \\
\hline Dyskinesia & $57(6.4)$ \\
\hline Polyuria and polydipsia & $31(3.5)$ \\
\hline
\end{tabular}

\subsection{Histopathological classification and location}

According to the basic classification of tumor histopathology defined in the 2016 WHO classification of CNS tumors, there were 180 cases of diffuse astrocytoma and oligodendrocyte tumors (ODs), which is the most common, accounting for $27.7 \%$. A total of 128 embryonal tumors (19.7\%), 116 tumors of the sellar region (17.8\%), 49 germ cell tumors (7.5\%), 47 mesenchymal, non-meningothelial tumors (7.2\%), and 41 ependymal tumors (6.3\%). Less common types were neuronal and mixed neuronal-glial tumors (3.5\%), followed by tumors of the pineal region (2.3\%), tumors of the cranial and paraspinal nerves (2.2\%), and meningiomas (1.8\%) (Fig. 2). A total of 413 $(63.5 \%)$ brain lesions were found in the supratentorial area and $237(36.5 \%)$ in the infratentorial area. The ratio of supratentorial to infratentorial was 1.74:1 (Table 2). The detailed tumor classification and location data are shown in Supplementary Table 1. 
Table 2

\begin{tabular}{|} 
Location of children's intracranial tumors \\
\hline Location & $\mathrm{n}(\%)$ \\
\hline Supratentorial & $405(62.3)$ \\
\hline Sellar region & $173(26.6)$ \\
\hline Lateral ventricle & $27(4.2)$ \\
\hline Cerebral hemisphere & $183(28.2)$ \\
\hline third ventricle & $8(1.2)$ \\
\hline pineal region & $14(2.1)$ \\
\hline Infratentorial & $245(37.7)$ \\
\hline fourth ventricle. & $117(18.0)$ \\
\hline brainstem & $21(3.2)$ \\
\hline cerebellum & $107(16.5)$ \\
\hline
\end{tabular}

\subsection{Grade}

Low-grade tumors (WHO I/II) constituted 60.9\%(396 cases) of all cases, while the rest were high-grade tumors (WHO III/IV) (254 cases, $39.1 \%$ ). The ratio of the two was 1.29:1. The statistics of tumor grade and children's age showed that the majority of high-grade and lowgrade tumors were children aged 10-15 years, and the majority of tumors in all age groups were low-grade (Fig. 3)

\subsection{The top five common intracranial tumors in children}

In our data, the top five tumors with the highest incidence rate constituted more than half of childhood brain tumors: astrocytomas (all subtypes) in $110(16.9 \%)$ patients, medulloblastoma in 88(13.5\%), craniopharyngioma in 83(12.8\%), ependymoma in 41(6.3\%), and germinoma in 38(5.8\%), as shown in Table 3. In terms of tumor grade, astrocytomas and craniopharyngiomas were mostly low-grade, medulloblastoma and germ cell tumors were mainly high-grade, and no significant difference in the grade of ependymoma. The top five common tumors were mostly low-grade, with a ratio of 1.28:1. Sex distribution showed that except for craniopharyngioma, the majority of other types of tumors were male children, and the majority of craniopharyngiomas were female children. Among the top five common tumors, 204 of the case were male and 154 were female, with a male to female ratio of 1.32:1. Regarding the location of tumor distribution, there was no significant difference in the location of astrocytomas, ependymoma was mostly subtentorial, and germinomas were mostly supratentorial. Among the top five common tumors, 190 were located in the supratentorial region, and 170 were subtentorial, with a ratio of 1.12:1.

Table 3

Grade, sex and location distribution of the most common five tumors

\begin{tabular}{|lllllllll|}
\hline \multirow{2}{*}{$\begin{array}{l}\text { Tumor } \\
\text { classification }\end{array}$} & $\mathrm{n}(\%)$ & $\begin{array}{l}\text { average } \\
\text { age }\end{array}$ & & Sex & \multicolumn{3}{c|}{ Grade } & \multicolumn{2}{l|}{ Location } \\
\cline { 5 - 9 } & & & Male(\%) & Female(\%) & Low(\%) & High(\%) & Supratentorial(\%) & Subtentorial(\%) \\
\hline Astrocytoma & $110(16.9)$ & 8.5 & $60(9.2)$ & $50(7.7)$ & $99(15.2)$ & $11(1.7)$ & $54(8.3)$ & $56(8.6)$ \\
\hline Medulloblastoma & $88(13.5)$ & 7.3 & $57(8.8)$ & $31(4.8)$ & - & $88(13.5)$ & - & $88(13.5)$ \\
\hline Craniopharyngioma & $83(12.8)$ & 8.7 & $41(6.3)$ & $42(6.5)$ & $83(12.8)$ & - & $83(12.8)$ & - \\
\hline Ependymoma & $41(6.3)$ & 6.9 & $22(3.4)$ & $19(2.9)$ & $20(3.0)$ & $21(3.2)$ & $16(2.4)$ & $25(3.8)$ \\
\hline Germinoma & $38(5.8)$ & 10.8 & $24(3.7)$ & $14(2.2)$ & $2(0.3)$ & $36(5.5)$ & $37(5.7)$ & $1(0.2)$ \\
\hline
\end{tabular}

\subsection{The most common subtype intracranial tumors in children}


In 110 patients with astrocytomas (all subtypes), there were 62 cases of PA, which is the most common, accounting for $61 \%$ (Fig. 4). There were 15 cases of diffuse astrocytoma (14\%), 10 cases of oligoastrocytoma (10\%), 7 cases of anaplastic astrocytoma (7\%), 5 cases of pleomorphic xanthoastrocytoma (5\%), and 3 cases of anaplastic pleomorphic xanthoastrocytoma (3\%). PA is the most common intracranial tumor subtype in children; therefore, we used it as the follow-up research object.

\subsection{Identification of DEGs in PA}

For the GSE44971 dataset, we identified a total of 3264 DEGs (489 upregulated genes and 2775 downregulated genes) between PA tumors and normal cerebellum samples (Supplementary Table 2). The top 10 significantly expressed genes were RTP4, C16orf54, LTF, IGJ, DKFZp667F0711, SLC35F4, LHX1, OLFM3, BC040734, and INSM1. Among them, the first five were upregulated genes and the following five were downregulated genes (Fig. 5A). The heatmap displays the expression profiles of these DEGs (Fig. 5B).

\subsection{The molecular mechanisms of DEGs}

To dissect the potential biological functions of 3264 DEGs in PA, we performed GO, DO, and GSEA analyses. The top 10 GO terms (BP, CC, and MF) are depicted in Fig. 6A, from which we found that DEGs mainly participated in the process of neural signal transduction. Of note, DEGs were also significantly correlated with several immune-related biological processes, such as regulation of leukocyte proliferation, regulation of lymphocyte proliferation, humoral immune response, and macrophage chemotaxis (Supplementary Table 3). DO enrichment analysis suggested that DEGs were highly associated with 'lung disease', 'periodontal disease', 'skin disease', and 'obesity' (Fig. 6B and Supplementary Table 4). The results of GSEA showed that DEGs were only markedly enriched in 5 KEGG signaling pathways, including 'neuroactive ligand receptor interaction', 'olfactory transduction', 'basal cell carcinoma', 'hedgehog signaling pathway', and 'taste transduction' (Fig. 6C and Supplementary Table 5).

\subsection{DNA methylation analysis in the GSE44684}

We obtained a total of 36787 differentially methylated sites between normal cerebellum and PA tumor samples in the GSE44684 dataset, of which 14924 were hypermethylated sites $(\Delta \beta>2)$ and 21863 were hypomethylated sites $(\Delta \beta<2)$ (Fig. 7A and Supplementary Table 6). The results of all differentially methylated sites from each autosomal chromosome are shown in the outer circle of the circos plot. The two innermost circles in the circos plot displayed differential hypermethylation and hypomethylation frequencies in a $10 \mathrm{Mb}$ sliding window across the genome (Fig. 7B). The pyramid plot showed the frequencies of differential hypermethylation and hypomethylation in each chromosome (Fig. 7C). Subsequently, we further examined the correlations between promoter DNA methylation and corresponding gene expression, and a total of 45 DEGs were identified for further analysis (Supplementary Fig. 1 and Supplementary Table 7).

\subsection{Identification of characteristic genes in PA}

To screen for biomarkers of PA from the 45 DEGs, we adopted the LASSO and SVM-RFE algorithms. Eight characteristic genes (NCKAP1L, GPR37L1, CSPG4, PPFIA4, CD86, CD1D, SLC15A3, and C8orf46) were identified using the LASSO algorithm (Fig. 8A-B). A total of 20 characteristic genes were selected using the SVM-RFE algorithm, as shown in Fig. 8C and Supplementary Table 8. After that, using the Venn diagram(Fig. 8D), we obtained five hub genes by overlapping the characteristic genes screened by the two algorithms, namely Nckassociated protein 1-like (NCKAP1L), G protein-coupled receptor 37-like 1 (GPR37L1), chondroitin sulfate proteoglycan 4 (CSPG4), chondroitin sulfate proteoglycan-4 (PPFIA4), and chromosome 8 open reading frame 46 (C8orf46). Except for PPFIA4, the other four hub genes were highly expressed in PA.

\subsection{Diagnostic capacity of hub genes}

We evaluated the diagnostic value of five hub genes in the GSE44971 dataset using ROC analysis. The results indicated that these genes had a powerful ability to discriminate PA from normal samples. The AUC of GPR37L1 was 0.994, and the AUC of the other genes was 1.000. Specific information is displayed in Fig. 9A-E.

\subsection{Correlation of the five hub genes with immune infiltration}

Previous results have shown that DEGs were markedly enriched in BP terms related to the immune system. Studies have shown that the TME plays a significant role in suppressing or enhancing the immune response. Consequently, we investigated the association between the expression levels of five hub genes and the TME. As shown in Fig. 10A-C and Supplementary Fig. 2, the immune, stromal, and estimate scores in the NCKAP1L high expression group were higher than those in the NCKAP1L low expression group. Unfortunately, there was no significant correlation between the other four hub genes (GPR37L1, CSPG4, PPFIA4, and C8orf46) and TME. Subsequently, 
SSGSEA analysis revealed a difference in the abundance of 28 immune-related gene sets in normal and PA tumor samples. Compared with the normal group, the PA tumor group had a higher proportion of immune gene sets, except activated CD4 T cells and effector memory CD4 T cells (Fig. 10D). We further evaluated the correlation between the expression of NCKAP1L and immune gene sets. Interestingly, NCKAP1L was found to be positively correlated with all immune gene sets, especially in macrophages (Fig. 10E).

\section{Discussion}

Intracranial tumors in children are not the comparable with those of adults, and also have obvious differences in terms of topographical distribution, pathological types, treatment, prognosis, and outcome. Consistent with the vast majority of other studies in the Asian region(Ahmed et al. 2007, Cho et al. 2002, Makino et al. 2010, Wong et al. 2005), our study also revealed a predominance of boys with intracranial tumors, with a sex ratio of 1.41:1, which was slightly higher than that in Western countries(Bauchet et al. 2009, El-Gaidi 2011, Hjalmars et al. 1999). Tumors at different life stages reflect different dynamics and biological behaviors(Rickert \& Paulus 2001). In our study, the mean age of the affected children was 8.8, which is similar to the results of studies in Iran and Pakistan(Ahmed et al. 2007, Mehrazin \& Yavari 2007). Our study suggested that the number of patients increased steadily with age and peaked at the age of 10-15 years. This phenomenon was also reported in a study conducted at the Beijing Neurosurgical Institute, Beijing, China(Zhou et al. 2008), and the findings were corroborated by studies in England, Sweden, and India(C et al. 2016, Govindan et al. 2018, Stiller et al. 2019). Conversely, some studies have suggested that the highest incidence occurs in the 5-9-year age group(Cho et al. 2002, Makino et al. 2010, Riaz et al. 2019). We speculated that this may be caused by the shyness of children in western China to express their symptoms, and when they grow up, they are more able to complain about their headache, visual disturbances, or endocrinopathies such as increased thirst or urination.

Regardless of tumor location, intracranial hypertension (53.9\%) and ocular symptoms (11.7\%) were the main clinical manifestations in our cohort of patients. However, our study also highlighted several other symptoms that indicate the possibility of developing tumors, such as weight loss, behavioral changes, school difficulties, developmental delay, head tilt, macrocephaly, diabetes insipidus, abnormal menstruation, and growth arrest. Furthermore, we were also concerned about the fact that 81 patients $(9.1 \%)$ were asymptomatic before diagnosis. Hence, improving the comprehensive examination of children and raising awareness of the various complex and atypical symptoms of brain tumors in children will help in diagnosing the tumor and in alleviating the suffering of the child expeditiously.

In this study, we followed the WHO Classification of Central Nervous System Tumors 2016 for the diagnosis of different tumors. As for the most common intracranial tumors in children, most studies have reported that astrocytoma followed by medulloblastoma are the two most common intracranial tumors(Aghadiuno et al. 1985, El-Gaidi 2011, Katchy et al. 2013, King et al. 2015, Lannering et al. 2009, Ogun et al. 2016, QT et al. 2020, Rickert \& Paulus 2001, Shanmugavadivel et al. 2020, Udaka \& Packer 2018). However, some studies have reported medulloblastomas to be the most common pediatric intracranial tumors ahead of astrocytomas(Almutrafi et al. 2020, Govindan et al. 2018, Kadri et al. 2005, Nasir et al. 2010, Shah et al. 2015). According to the present study, astrocytomas with a frequency of $16.9 \%$ were the most common brain tumors, with the average age of onset of this tumor being 8.5 years. Among the 110 patients, there was a slight male predominance, with a ratio of 1.2:1, and there was no specific location preference. Obviously, most astrocytomas belong to low grade tumors, which is consistent with previous research(El-Gaidi 2011, Riaz et al. 2019, Shah et al. 2015). Medulloblastoma, an embryonal tumor of the posterior fossa, comprises up to $20 \%$ of all pediatric brain tumors(Ostrom et al. 2019, Shtil 2016). In our study, medulloblastoma was the second most common brain tumor, accounting for $13.5 \%$ of all brain tumors with a male predominance (ratio of 1.8). It has two incidence peaks in patients aged $4-5$ years (18\%) and in patients aged $7-8$ years $(23.8 \%)$ with a mean age of 7.8 years, which is slightly different from previous studies(Udaka \& Packer 2018) but consistent with the study in China(Zhou et al. 2008). Craniopharyngioma is a benign epithelial tumor with slow growth that accounts for approximately $5-10 \%$ of pediatric brain tumors(Garre \& Cama 2007, Muller et al. 2019). In this study, craniopharyngioma, the third most common, had a mean age of onset of 8.7 years and no discrepancy in incidence between sexes. Moreover, the critical location of the lesion and the difficulty in treating postoperative complications make the prognosis of patients generally poor(Muller et al. 2006). In our follow-up results of the 83 pediatric craniopharyngioma patients in our study, 10 patients died and 18 relapsed. Ependymomas are the fourth most common type, accounting for $6.3 \%$ of all pediatric brain tumors with a mean age of 6.9 years, consistent with some studies in Asia and Europe(C et al. 2016, El-Gaidi 2011, Makino et al. 2010, Zhou et al. 2008). Our study showed that there was a subtentorial predominance with a ratio of $1.56: 1$ and a slightly male predominance, with a ratio of 1.16:1. Germinoma, which accounts for $5.8 \%$ of all brain tumors in children, is the fifth most common, consistent with the consensus(Fetcko \& Dey 2018).

Consistent with previous studies(Riaz et al. 2019), pilocytic astrocytomas were the most common childhood brain tumor subtypes in the present study. Studies have shown that pilocytic astrocytoma is associated with high levels of myeloid and lymphocyte infiltration and activation marker expression(Forsyth et al. 1993, Huang et al. 2005, Rosemberg \& Fujiwara 2005). Therefore, it is inferred that immune

Page $7 / 17$ 
cells are related to the occurrence and development of pilocytic astrocytomas. Based on the incidence rate of this type of tumor and the current treatment methods that are still not perfect, we decided to carry out bioinformatics analysis to provide theoretical guidance for further research.

In the present study, by analyzing the GE044971 dataset, we identified 3264 DEGs in the tumor tissue. We then performed GO, DO, and GSEA analyses to explore the biological functions of the DEGs. The results showed that DEGs participated in the functions of neurotransmitter transfer and transmembrane transport, and are highly associated with lung disease, periodontal disease, skin disease, and obesity, and markedly enriched in neuroactive ligand receptor interaction, olfactory transduction, basal cell carcinoma, hedgehog signaling pathyway, and taste transduction KEGG pathways. Notably, immune-related functional items were found in the GO-BP pathway. These results suggest that DEGs were highly correlated with the immune system, which confirms our hypothesis.

DNA methylation causes transcriptional silencing to regulate gene expression. Therefore, we carried out methylation analysis on the GSE44864 dataset to determine whether methylation affects gene expression in pilocytic astrocytoma, and further precise exploration of differential genes. The results showed that methylation had the greatest effect on the expression of chromosome 17. The results of methylation site analysis were correlated with the gene expression of corresponding samples, and 45 genes with negative regulation of promoter methylation level and gene expression level were screened out. Therefore, the differential expression of some differential genes may be due to differential methylation of their own genes.

To screen the hub genes among these 45 differentially expressed genes, we used LASSO regression and the SVM-RFE algorithm. At the intersection of the two algorithms, we obtained five hub genes: NCKAP1L, GPR37L1, CSPG4, PPFIA4, and C8orf46, and their efficiency was also confirmed by the ROC curve.

In recent years, an increasing number of studies have focused on TME, in which immune cells and stromal cells play an important role in the diagnosis and prognosis of tumors(Hanahan \& Coussens 2012). Therefore, we first performed ssGSEA analysis on the GSE44971 dataset. We found that compared with the normal group, the PA tumor group had a higher proportion of immune gene sets, except for activated CD $4 \mathrm{~T}$ cells and effector memory CD4 T cells. This result suggests that we should perform further immunological analysis of the five hub genes. Then, using the ESTIMATE algorithm, we found that the high and low expression groups of NCKAP1L gene had significant differences in the immune, stromal, and estimate scores. Consequently, we further evaluated the correlation between the expression of NCKAP1L and immune gene sets, and found that this gene was positively correlated with all immune gene sets, especially in macrophages.

Our study is the first to report the relationship between NCKAP1L and PA. NCKAP1L, or Nck-associated protein 1-like, is a key component of the actin cytoskeleton machinery. The protein encoded by this protein is hematopoietic protein-1 (HEM-1), a hematopoietic lineagerestricted member of the Nap1l subunit of the WAVE (WASP-family verprolin-homologous protein) complex(Weiner et al. 2006). The role of this gene in tumors has not been extensively studied. Recently, it has been found that this gene plays an important role in the activation, migration, and cell contact formation of lymphoid and myeloid cells, including the formation of immune synapses in effector cells(Park et al. 2008). Subsequently, a few studies have revealed that it is associated with immunodeficiency, lymphoproliferation, and high inflammation, and may be a biomarker of some tumors(CN et al. 2020, M et al. 2020, Y et al. 2020). Similarly, our study also found that as a hub gene of PA, NCKAP1L plays an important role in the immune system. It is closely related to $B$ cells and macrophages and may affect the TME in this way, thereby affecting the development of PA. Therefore, we provide a new basis and direction for the pathogenesis of PA and the study of the TME.

G protein-coupled receptor 37-like 1 (GPR37L1) and chondroitin sulfate proteoglycan 4 (CSPG4) have been shown to be expressed in astrocytomas and gliomas. GPR37L1 interacts with patched 1 (Ptch1) in the peri-ciliary membranes of astrocytes(La Sala et al. 2020), while CSPG4, a type I transmembrane protein, plays a role in the origin, progression, and angiogenesis of glioma(Mellai et al. 2020). Both these genes play a role in TME and may be promising as biomarkers for detecting PA progression and patient survival(Mellai et al. 2020). PPFIA4, also known as LIP.1 or Liprin alpha1, encodes liprin, which is related to neural signal transmission. Previous studies have shown that it is correlated with pancreatic cancer and small cell lung cancer(BA et al. 2009). Our study found that it may be one of the characteristic biomarkers of PA, and further research is needed to explore its role in tumor development and its relationship with TME. Chromosome 8 open reading frame 46 (C8orf46), a human protein-coding gene, has been named Vexin. Previous studies have suggested that Vexin is involved in embryonic neurogenesis and is related to cancer progression, but its function has not yet been elucidated clearly (Koshimizu et al. 2020, Moore et al. 2018). Our study is the first to report that this gene is associated with PA, which provides a new direction for future research. 


\section{Conclusion}

The present analysis, based on a large series of pediatric patients, provides a reliable profile of the epidemiology of nervous system tumors in children and valuable information for worldwide epidemiological research. We found that PA was the most common tumor subtype in childhood brain tumors. Then, through bioinformatics analysis, we suggested that NCKAP1L, GPR37L1, CSPG4, PPFIA4, and C8orf46 are potential biomarkers for the diagnosis of PA. NCKAP1L plays an important role in the immune system and may affect the TME.

The findings of this study must be considered in light of some limitations. First, the data of this study were obtained from a single center of pediatric neurosurgery in Northwest China, which may cause possible bias in the population source. Second, five hub genes were not tested in human tumor tissues. Therefore, we should verify the results and further explore the value of key genes in the diagnosis and treatment of PA. We are looking forward to multi-center and multidisciplinary collaborative research to improve the prognosis of children with intracranial tumors in China and other countries by establishing reasonable diagnosis, treatment systems, and evaluation standards for intracranial tumors, and perfecting the epidemiological follow-up system.

\section{Declarations}

\section{Funding}

The present work was supported by the Natural Science Foundation of China (NSFC) grant to Xiaosheng He (Nos. 81971156 and 2020XC015).

\section{Author Contributions}

GW had the idea for the article; GW, YJ designed the experiments; GW, YJ, and EK carried out the experiments. GW and HC analyzed the data and experimental results. The manuscript was written by GW and YY. YJ and JW critically revised the work. All authors have read and approved the final manuscript.

\section{Data Availability}

The datasets generated and analyzed during the current study are available from the corresponding author on reasonable request. The data and materials underlying this article are available in the article.

\section{Availability of data and materials}

Not applicable.

\section{Ethics approval and consent to participate}

The collection of the patient samples, the isolation of tumor cells, and the analysis of the obtained samples was approved by the ethics committee of Xijing Hospital(KY20202060-F-1). Our study adhere to the tenets of the 1964 Declaration of Helsinki. All studies obtained informed sympathy from patients.

\section{Consent for publication}

Not applicable.

\section{Competing interests}

The authors declare that they have no competing interests.

\section{Acknowledgements}

Not applicable.

\section{References}


1. Aghadiuno PU, Adeloye A, Olumide AA, Nottidge VA (1985): Intracranial neoplasms in children in Ibadan, Nigeria. Childs Nerv Syst 1 , 39-44

2. Ahmed N, Bhurgri Y, Sadiq S, Shakoor KA (2007): Pediatric brain tumours at a tertiary care hospital in Karachi. Asian Pac J Cancer Prev 8, 399-404

3. Almutrafi A, Bashawry Y, AlShakweer W, Al-Harbi M, Altwairgi A, Al-Dandan S (2020): The Epidemiology of Primary Central Nervous System Tumors at the National Neurologic Institute in Saudi Arabia: A Ten-Year Single-Institution Study. J Cancer Epidemiol 2020, 1429615

4. Anonymous (2006): The Gene Ontology (GO) project in 2006. NUCLEIC ACIDS RES 34, D322-6

5. BA D, J J, H S, GH S, DJ W, CH D, M S, GL C, K C (2009): A novel mechanism of hippocampal LTD involving muscarinic receptortriggered interactions between AMPARs, GRIP and liprin-alpha. MOL BRAIN 2, 18

6. Barbie DA et al. (2009): Systematic RNA interference reveals that oncogenic KRAS-driven cancers require TBK1. NATURE 462, 108-12

7. Bauchet L, Rigau V, Mathieu-Daude H, Fabbro-Peray P, Palenzuela G, Figarella-Branger D, Moritz J, Puget S, Bauchet F, Pallusseau L, Duffau H, Coubes P, Tretarre B, Labrousse F, Dhellemmes P (2009): Clinical epidemiology for childhood primary central nervous system tumors. J Neurooncol 92, 87-98

8. Bindea G, Mlecnik B, Tosolini M, Kirilovsky A, Waldner M, Obenauf AC, Angell H, Fredriksen T, Lafontaine L, Berger A, Bruneval P, Fridman WH, Becker C, Pages F, Speicher MR, Trajanoski Z, Galon J (2013): Spatiotemporal dynamics of intratumoral immune cells reveal the immune landscape in human cancer. IMMUNITY 39, 782-95

9. C E, I K, G A, O C, M D, S L, B S (2016): Clinical characteristics and late effects in CNS tumours of childhood: Do not forget long term follow-up of the low grade tumours. European journal of paediatric neurology : EJPN : official journal of the European Paediatric Neurology Society 20, 580-7

10. Chen F, Zhuang X, Lin L, Yu P, Wang Y, Shi Y, Hu G, Sun Y (2015): New horizons in tumor microenvironment biology: challenges and opportunities. BMC MED 13, 45

11. Cho KT, Wang KC, Kim SK, Shin SH, Chi JG, Cho BK (2002): Pediatric brain tumors: statistics of SNUH, Korea (1959-2000). Childs Nerv Syst 18, 30-7

12. CN C et al. (2020): NCKAP1L defects lead to a novel syndrome combining immunodeficiency, lymphoproliferation, and hyperinflammation. The Journal of experimental medicine 217

13. Dabiao Z, Shiqi L, Zhenyu M (2007): Epidemiology of nervous system tumors in childhood: a survey based on 1267 consecutive cases. CHINESE JOURNAL OF NEUROSURGERY 23, 4-7

14. El-Gaidi MA (2011): Descriptive epidemiology of pediatric intracranial neoplasms in Egypt. PEDIATR NEUROSURG 47, 385-95

15. Fetcko K, Dey M (2018): Primary Central Nervous System Germ Cell Tumors: A Review and Update. Med Res Arch 6

16. Finlay JL, Goldman S, Wong MC, Cairo M, Garvin J, August C, Cohen BH, Stanley P, Zimmerman RA, Bostrom B, Geyer JR, Harris RE, Sanders J, Yates AJ, Boyett JM, Packer RJ (1996): Pilot study of high-dose thiotepa and etoposide with autologous bone marrow rescue in children and young adults with recurrent CNS tumors. The Children's Cancer Group. J CLIN ONCOL 14, 2495-503

17. Forsyth PA, Shaw EG, Scheithauer BW, O'Fallon JR, Layton DJ, Katzmann JA (1993): Supratentorial pilocytic astrocytomas. A clinicopathologic, prognostic, and flow cytometric study of 51 patients. CANCER-AM CANCER SOC 72, 1335-42

18. Friedman J, Hastie T, Tibshirani R (2010): Regularization Paths for Generalized Linear Models via Coordinate Descent. J STAT SOFTW 33, 1-22

19. Garre ML, Cama A (2007): Craniopharyngioma: modern concepts in pathogenesis and treatment. CURR OPIN PEDIATR 19, 471-9

20. Govindan A, Parambil RM, Alapatt JP (2018): Pediatric Intracranial Tumors over a 5-Year Period in a Tertiary Care Center of North Kerala, India: A Retrospective Analysis. Asian J Neurosurg 13, 1112-1117

21. Grivennikov SI, Greten FR, Karin M (2010): Immunity, inflammation, and cancer. CELL 140, 883-99

22. Hanahan D, Coussens LM (2012): Accessories to the crime: functions of cells recruited to the tumor microenvironment. CANCER CELL 21, 309-22

23. Hjalmars U, Kulldorff M, Wahlqvist Y, Lannering B (1999): Increased incidence rates but no space-time clustering of childhood astrocytoma in Sweden, 1973-1992: a population-based study of pediatric brain tumors. CANCER-AM CANCER SOC 85, 2077-90

24. Huang H, Hara A, Homma T, Yonekawa Y, Ohgaki H (2005): Altered expression of immune defense genes in pilocytic astrocytomas. J Neuropathol Exp Neurol 64, 891-901 
25. Huang ML, Hung YH, Lee WM, Li RK, Jiang BR (2014): SVM-RFE based feature selection and Taguchi parameters optimization for multiclass SVM classifier. ScientificWorldJournal 2014, 795624

26. Kaatsch P, Rickert CH, Kuhl J, Schuz J, Michaelis J (2001): Population-based epidemiologic data on brain tumors in German children. CANCER-AM CANCER SOC 92, 3155-64

27. Kadri H, Mawla AA, Murad L (2005): Incidence of childhood brain tumors in Syria (1993-2002). PEDIATR NEUROSURG 41, 173-7

28. Katchy KC, Alexander S, Al-Nashmi NM, Al-Ramadan A (2013): Epidemiology of primary brain tumors in childhood and adolescence in Kuwait. Springerplus 2, 58

29. King TZ, Na S, Mao H (2015): Neural Underpinnings of Working Memory in Adult Survivors of Childhood Brain Tumors. J Int Neuropsychol Soc 21, 494-505

30. Koshimizu H, Suzuki S, Kawai A, Miura R, Ohta KI, Miki T, Adachi N, Matsuoka H (2020): Vexin is upregulated in cerebral cortical neurons by brain-derived neurotrophic factor. Neuropsychopharmacol Rep 40, 275-280

31. Kuratsu J, Takeshima H, Ushio Y (2001): Trends in the incidence of primary intracranial tumors in Kumamoto, Japan. INT J CLIN ONCOL 6, 183-91

32. La Sala G, Di Pietro C, Matteoni R, Bolasco G, Marazziti D, Tocchini-Valentini GP (2020): Gpr37l1/prosaposin receptor regulates Ptch1 trafficking, Shh production, and cell proliferation in cerebellar primary astrocytes. J NEUROSCI RES

33. Lannering B, Sandstrom PE, Holm S, Lundgren J, Pfeifer S, Samuelsson U, Stromberg B, Gustafsson G (2009): Classification, incidence and survival analyses of children with CNS tumours diagnosed in Sweden 1984-2005. ACTA PAEDIATR 98, 1620-7

34. M H, J X, H H, M L, J W (2020): Prognostic Value of DNA Methylation-Driven Genes in Clear Cell Renal Cell Carcinoma: A Study Based on Methylation and Transcriptome Analyses. DIS MARKERS 2020, 8817652

35. Makino K, Nakamura H, Yano S, Kuratsu J (2010): Population-based epidemiological study of primary intracranial tumors in childhood. Childs Nerv Syst 26, 1029-34

36. Matthias, Karajannis, Jeffrey, C., Allen, Elizabeth, W., Newcomb (2008): Treatment of pediatric brain tumors. J CELL PHYSIOL

37. Mehrazin M, Yavari P (2007): Morphological pattern and frequency of intracranial tumors in children. Childs Nerv Syst 23, 157-62

38. Mellai M, Casalone C, Corona C, Crociara P, Favole A, Cassoni P, Schiffer D, Boldorini R (2020): Chondroitin Sulphate Proteoglycans in the Tumour Microenvironment. ADV EXP MED BIOL 1272, 73-92

39. Moore KB, Logan MA, Aldiri I, Roberts JM, Steele M, Vetter ML (2018): C8orf46 homolog encodes a novel protein Vexin that is required for neurogenesis in Xenopus laevis. DEV BIOL 437, 27-40

40. Muller HL, Albanese A, Calaminus G, Hargrave D, Garre ML, Gebhardt U, Saran F, Sorensen N, Spoudeas HA (2006): Consensus and perspectives on treatment strategies in childhood craniopharyngioma: results of a meeting of the Craniopharyngioma Study Group (SIOP), Genova, 2004. J Pediatr Endocrinol Metab 19 Suppl 1, 453-4

41. Muller HL, Merchant TE, Warmuth-Metz M, Martinez-Barbera JP, Puget S (2019): Craniopharyngioma. NAT REV DIS PRIMERS 5, 75

42. Nasir S, Jamila B, Khaleeq S (2010): A retrospective study of primary brain tumors in children under 14 years of age at PIMS, Islamabad. Asian Pac J Cancer Prev 11, 1225-7

43. Ogun GO, Adeleye AO, Babatunde TO, Ogun OA, Salami A, Brown BJ, Akang E (2016): Central nervous system tumours in children in Ibadan, Nigeria: a histopathologic study. Pan Afr Med J 24, 34

44. Osborne JD, Flatow J, Holko M, Lin SM, Kibbe WA, Zhu LJ, Danila MI, Feng G, Chisholm RL (2009): Annotating the human genome with Disease Ontology. BMC GENOMICS 10 Suppl 1, S6

45. Ostrom QT, Cioffı G, Gittleman H, Patil N, Waite K, Kruchko C, Barnholtz-Sloan JS (2019): CBTRUS Statistical Report: Primary Brain and Other Central Nervous System Tumors Diagnosed in the United States in 2012-2016. Neuro Oncol 21, v1-v100

46. Park H, Staehling-Hampton K, Appleby MW, Brunkow ME, Habib T, Zhang Y, Ramsdell F, Liggitt HD, Freie B, Tsang M, Carlson G, Friend S, Frevert C, Iritani BM (2008): A point mutation in the murine Hem1 gene reveals an essential role for Hematopoietic protein 1 in lymphopoiesis and innate immunity. J EXP MED 205, 2899-913

47. QT O, N P, G C, K W, C K, JS B (2020): CBTRUS Statistical Report: Primary Brain and Other Central Nervous System Tumors Diagnosed in the United States in 2013-2017. NEURO-ONCOLOGY 22, iv1-iv96

48. Quail DF, Joyce JA (2013): Microenvironmental regulation of tumor progression and metastasis. NAT MED 19, 1423-37

49. Riaz Q, Naeem E, Fadoo Z, Lohano M, Mushtaq N (2019): Intracranial tumors in children: a 10-year review from a single tertiary health-care center. Childs Nerv Syst 35, 2347-2353 
50. Rickert CH, Paulus W (2001): Epidemiology of central nervous system tumors in childhood and adolescence based on the new WHO classification. Childs Nerv Syst 17, 503-11

51. Ritchie ME, Phipson B, Wu D, Hu Y, Law CW, Shi W, Smyth GK (2015): limma powers differential expression analyses for RNAsequencing and microarray studies. NUCLEIC ACIDS RES 43, e47

52. Rosemberg S, Fujiwara D (2005): Epidemiology of pediatric tumors of the nervous system according to the WHO 2000 classification: a report of 1,195 cases from a single institution. Childs Nerv Syst 21, 940-4

53. Shah HC, Ubhale BP, Shah JK (2015): Demographic and histopathologic profile of pediatric brain tumors: A hospital-based study. South Asian J Cancer 4, 146-8

54. Shanmugavadivel D, Liu JF, Murphy L, Wilne S, Walker D (2020): Accelerating diagnosis for childhood brain tumours: an analysis of the HeadSmart UK population data. ARCH DIS CHILD 105, 355-362

55. Shtil AA (2016): Review on Oxford Textbook of Oncology. 3rd Edition, 2016. Recent Pat Anticancer Drug Discov 11,475

56. Solomon DA, Wood MD, Tihan T, Bollen AW, Gupta N, Phillips JJ, Perry A (2016): Diffuse Midline Gliomas with Histone H3-K27M Mutation: A Series of 47 Cases Assessing the Spectrum of Morphologic Variation and Associated Genetic Alterations. BRAIN PATHOL 26, 569-80

57. Steliarova-Foucher E, Stiller C, Kaatsch P, Berrino F, Coebergh JW, Lacour B, Parkin M (2004): Geographical patterns and time trends of cancer incidence and survival among children and adolescents in Europe since the 1970s (the ACCISproject): an epidemiological study. LANCET 364, 2097-105

58. Stiller CA, Bayne AM, Chakrabarty A, Kenny T, Chumas P (2019): Incidence of childhood CNS tumours in Britain and variation in rates by definition of malignant behaviour: population-based study. BMC CANCER 19, 139

59. Subramanian A, Tamayo P, Mootha VK, Mukherjee S, Ebert BL, Gillette MA, Paulovich A, Pomeroy SL, Golub TR, Lander ES, Mesirov JP (2005): Gene set enrichment analysis: a knowledge-based approach for interpreting genome-wide expression profiles. Proc Natl Acad Sci U S A 102, 15545-50

60. Suresh SG, Srinivasan A, Scott JX, Rao SM, Chidambaram B, Chandrasekar S (2017): Profile and Outcome of Pediatric Brain Tumors Experience from a Tertiary Care Pediatric Oncology Unit in South India. J Pediatr Neurosci 12, 237-244

61. Udaka YT, Packer RJ (2018): Pediatric Brain Tumors. NEUROL CLIN 36, 533-556

62. Villa C, Miquel C, Mosses D, Bernier M, Di Stefano AL (2018): The 2016 World Health Organization classification of tumours of the central nervous system. PRESSE MED 47, e187-e200

63. Weiner OD, Rentel MC, Ott A, Brown GE, Jedrychowski M, Yaffe MB, Gygi SP, Cantley LC, Bourne HR, Kirschner MW (2006): Hem-1 complexes are essential for Rac activation, actin polymerization, and myosin regulation during neutrophil chemotaxis. PLOS BIOL 4, e38

64. Wong TT, Ho DM, Chang KP, Yen SH, Guo WY, Chang FC, Liang ML, Pan HC, Chung WY (2005): Primary pediatric brain tumors: statistics of Taipei VGH, Taiwan (1975-2004). CANCER-AM CANCER SOC 104, 2156-67

65. Y W, M Z, F G, Y S, X F, G Q (2020): Identification of Tumor Microenvironment-Related Prognostic Biomarkers in Luminal Breast Cancer. FRONT GENET 11, 555865

66. Zhou D, Zhang Y, Liu H, Luo S, Luo L, Dai K (2008): Epidemiology of nervous system tumors in children: a survey of 1,485 cases in Beijing Tiantan Hospital from 2001 to 2005. PEDIATR NEUROSURG 44, 97-103

\section{Figures}


$30.00 \%$

$25.00 \%$

$20.00 \%$

$15.00 \%$

$10.00 \%$

$5.00 \%$

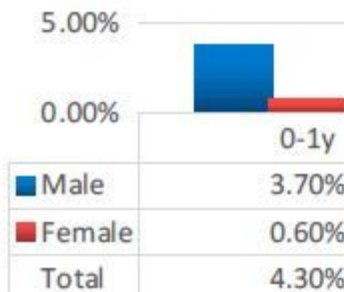

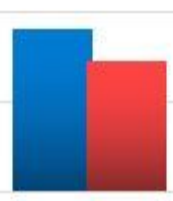

2-5y

$8.90 \%$

$7.10 \%$

$16.00 \%$
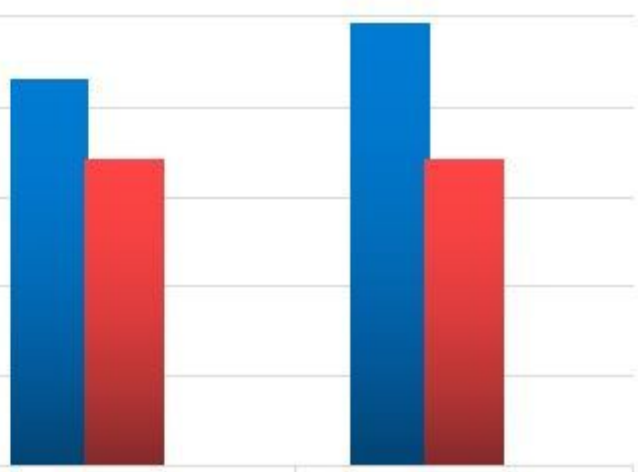

5-10y

10-15y

$21.40 \%$

$24.50 \%$

$16.90 \%$

$16.90 \%$

Total

$4.30 \%$

$38.30 \%$

$41.40 \%$

\section{Figure 1}

Sex and age distribution of children with intracranial tumors

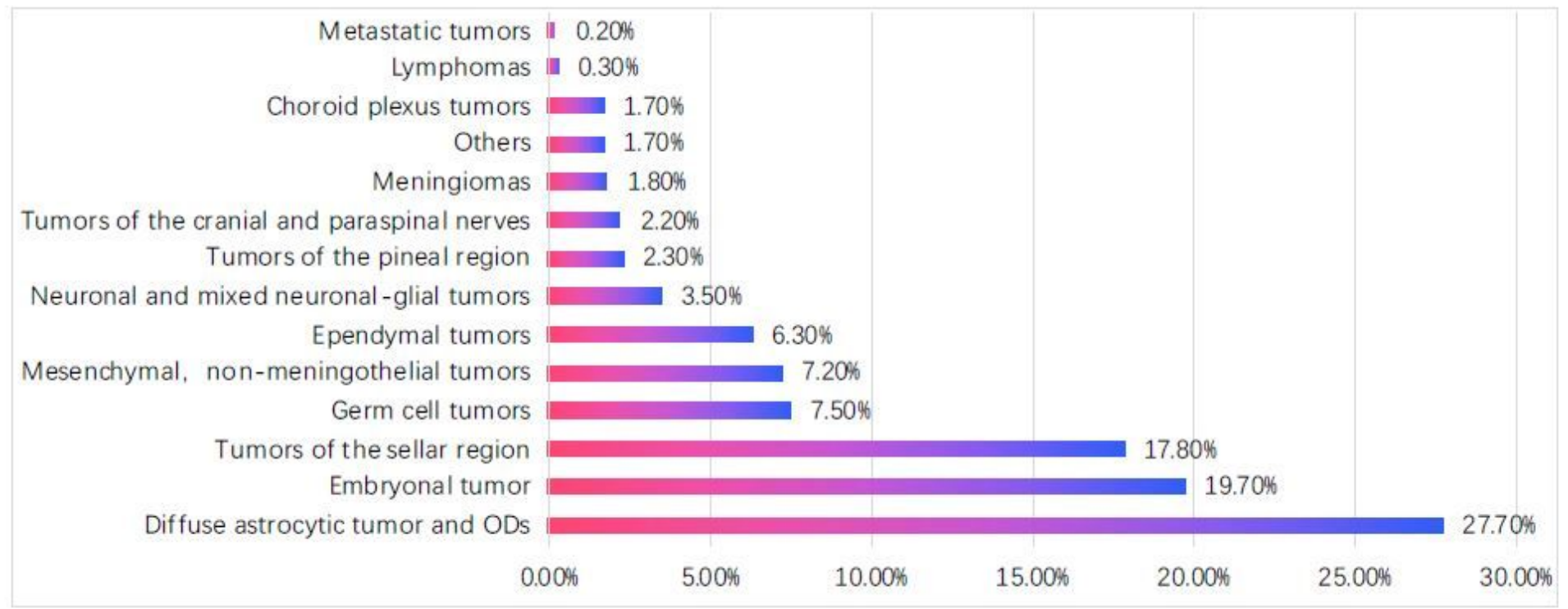

\section{Figure 2}

Basic pathological classification of intracranial tumor in children 


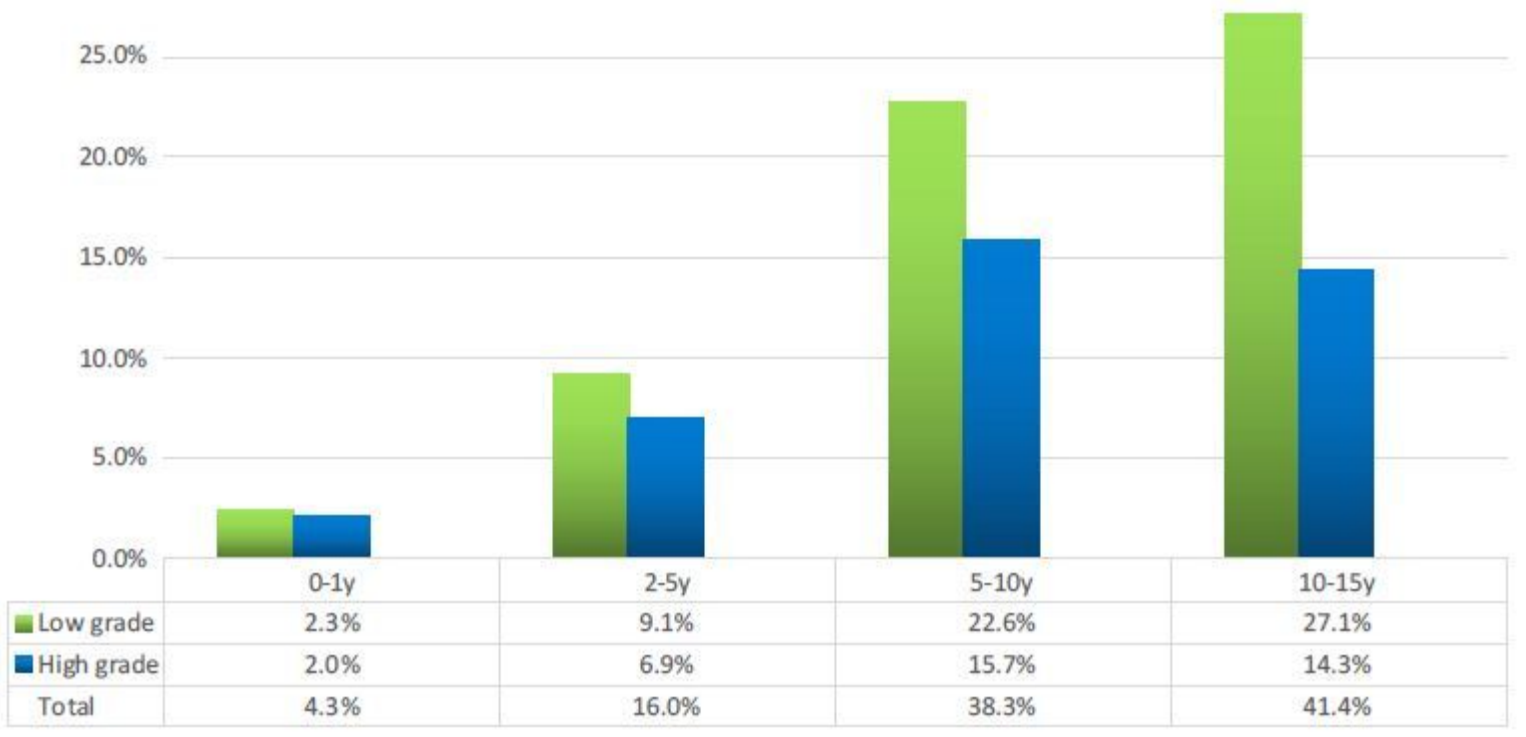

\section{Figure 3}

Grade and age distribution of children with intracranial tumor

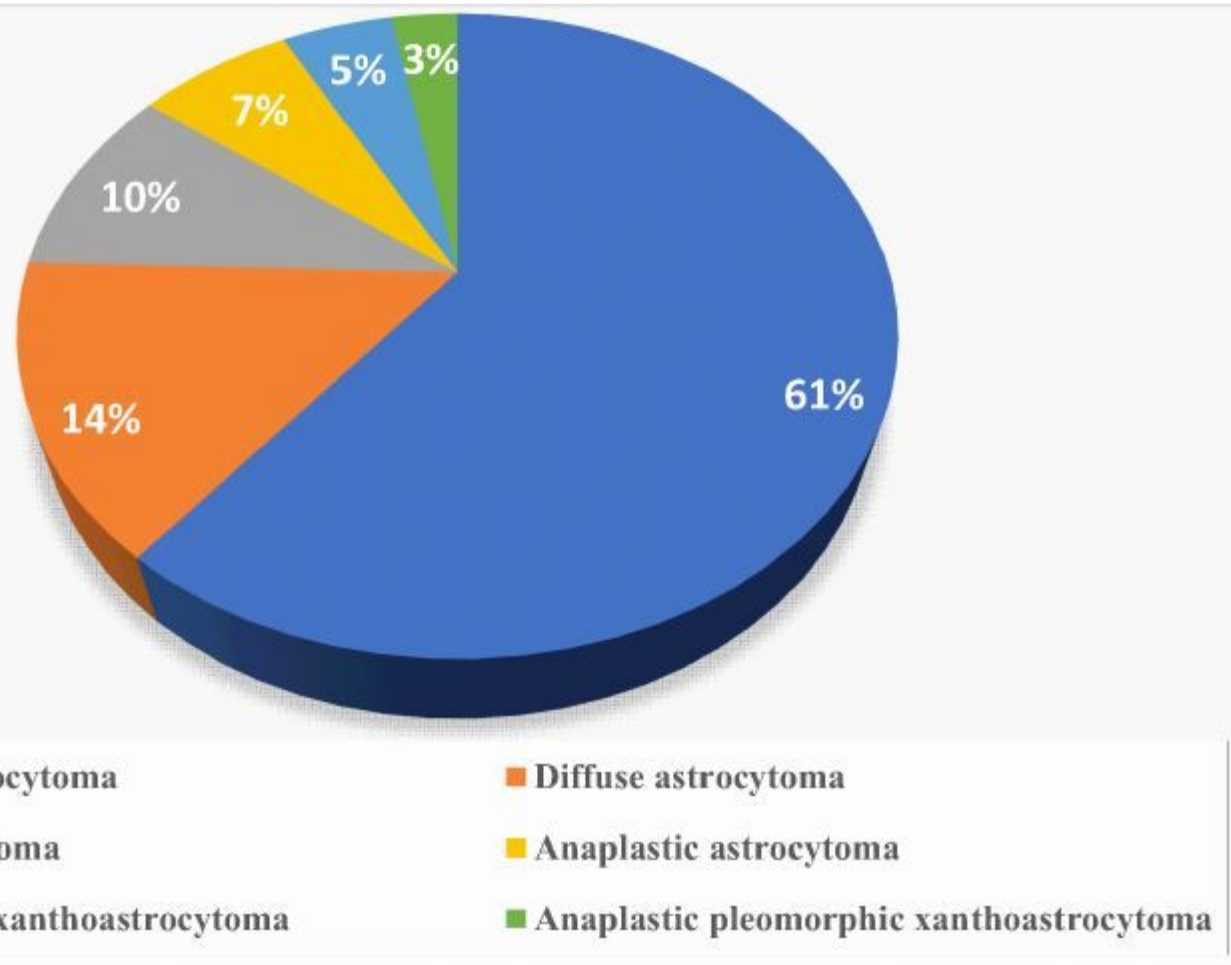

\section{Figure 4}

Distribution of astrocytoma 
A

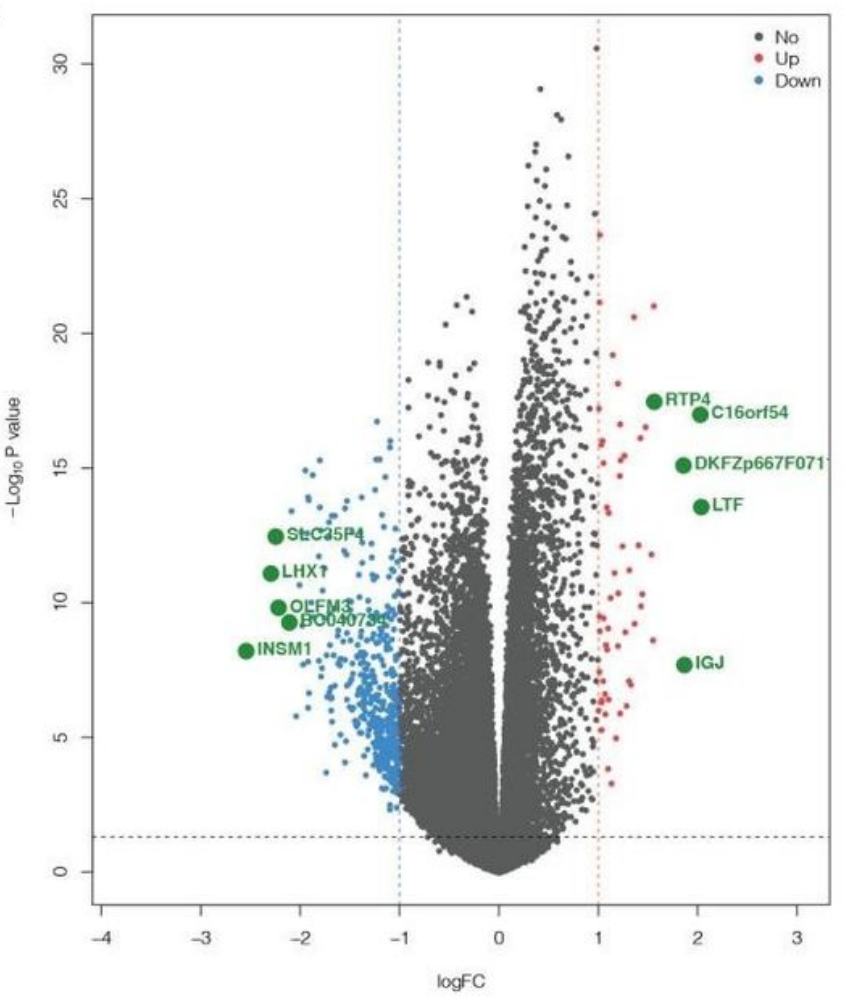

B

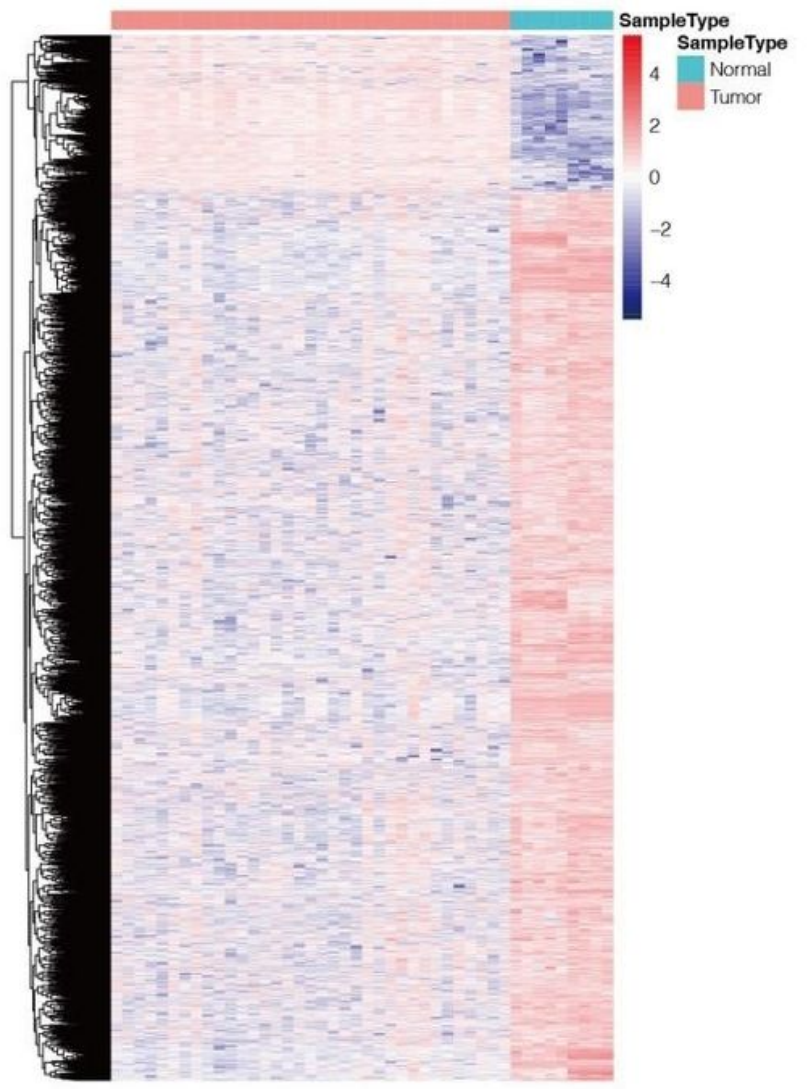

\section{Figure 5}

Identification of DEGs in PA. A: Volcano plot for DEGs between PA and normal cerebellum samples. B: Heatmap of DEGs.

\section{Figure 6}

GO, DO, and GSEA analyses. A: The top $10 \mathrm{GO}$ terms (BP, CC, and MF). B: DO enrichment analysis. B: The results of GSEA.

\section{Figure 7}

DNA methylation analysis. A: Volcano plot for the differentially methylated sites. B: Circos plot for the differentially methylated sites from each autosomal chromosome. C: Pyramid plot for the distribution of differentially methylated sites on chromosomes. 
A

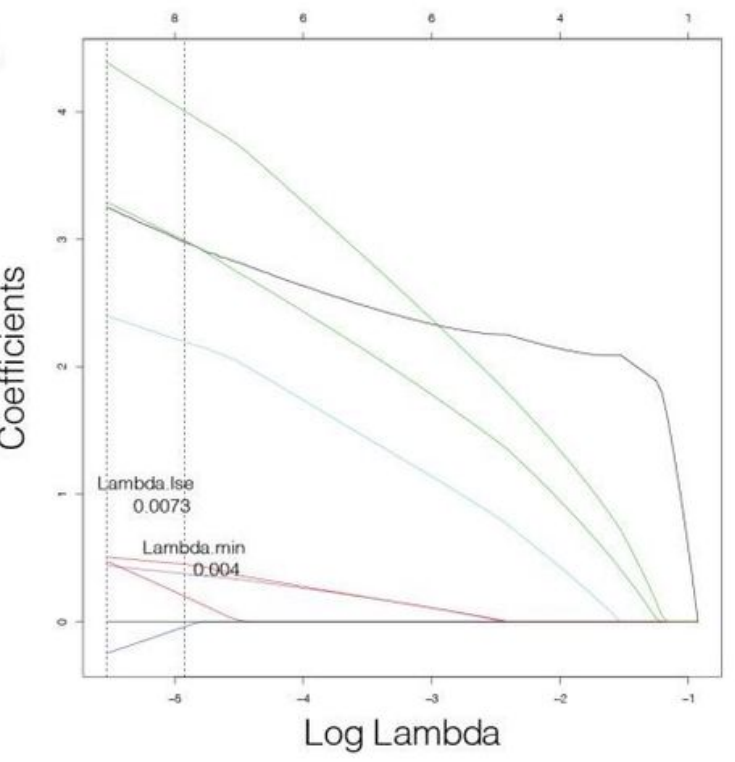

C

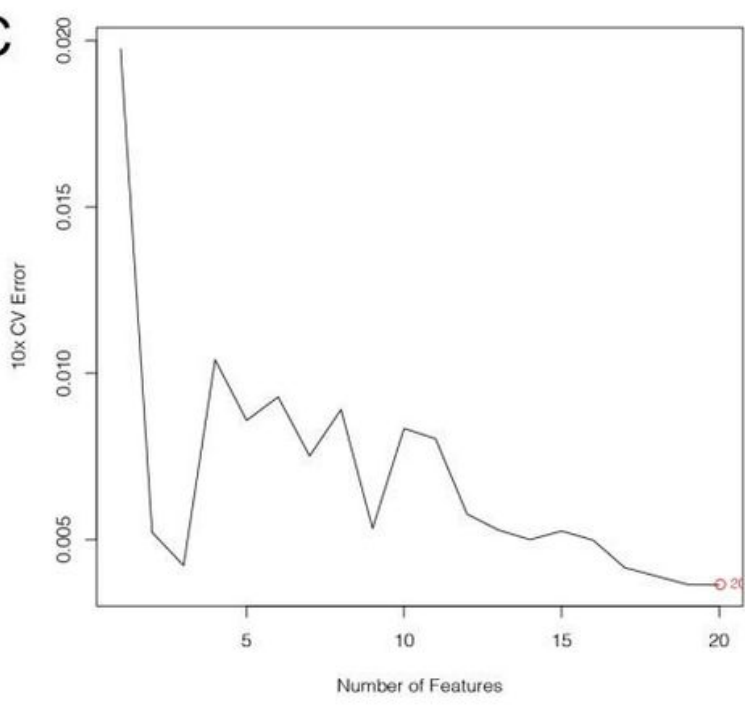

B

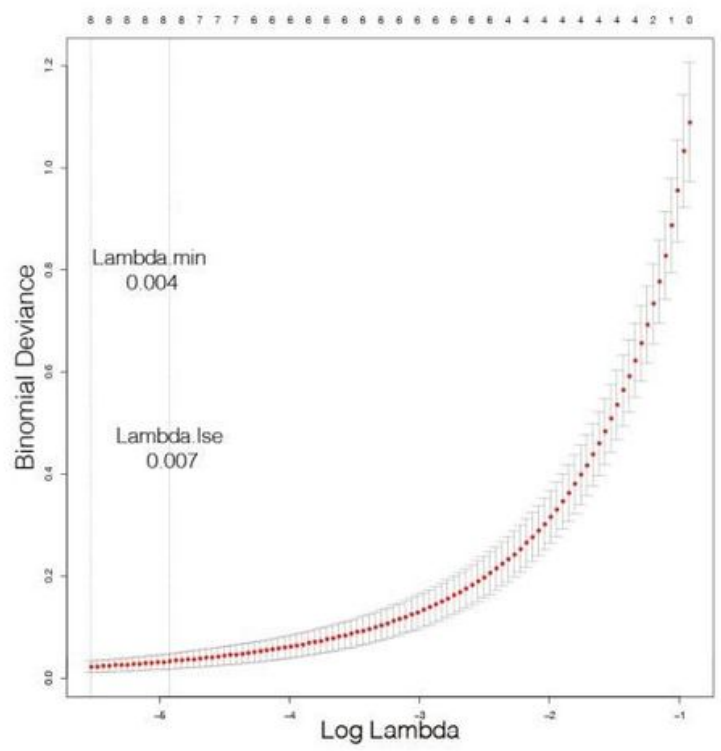

D

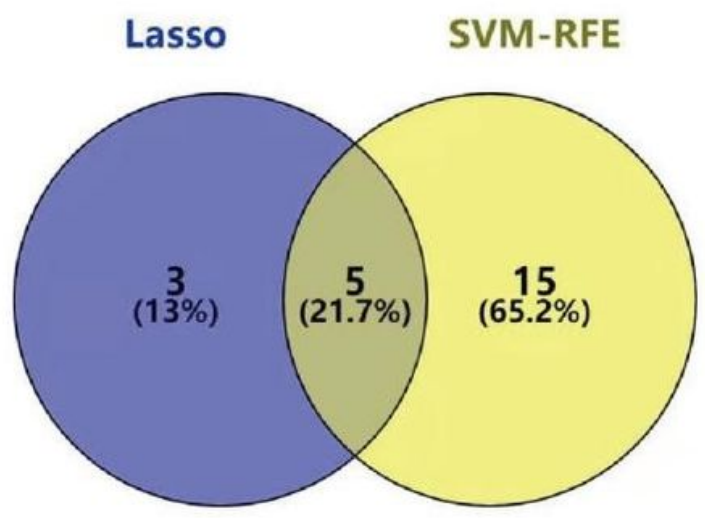

\section{Figure 8}

Identification of characteristic genes. A,B: Eight characteristic genes were discerned using the LASSO algorithm. C: Twenty characteristic genes were selected with the SVM-RFE algorithm. D: The Venn diagram of the characteristic genes identified by LASSO algorithm and SVM-RFE algorithm. We had obtained five hub genes. 
A

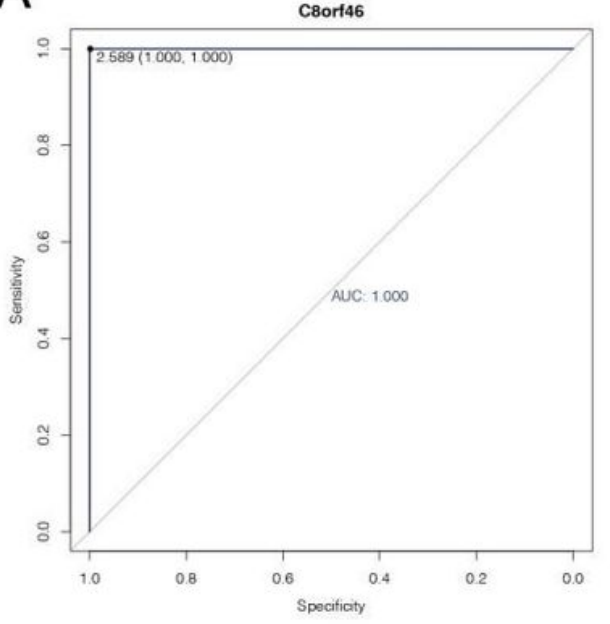

B

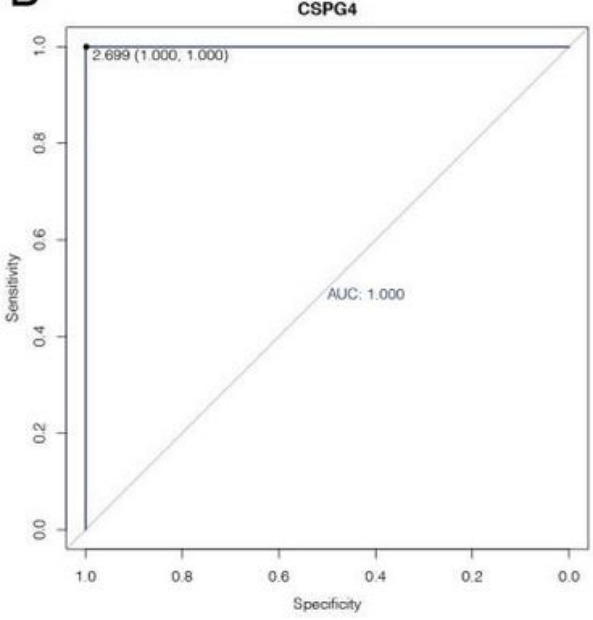

C

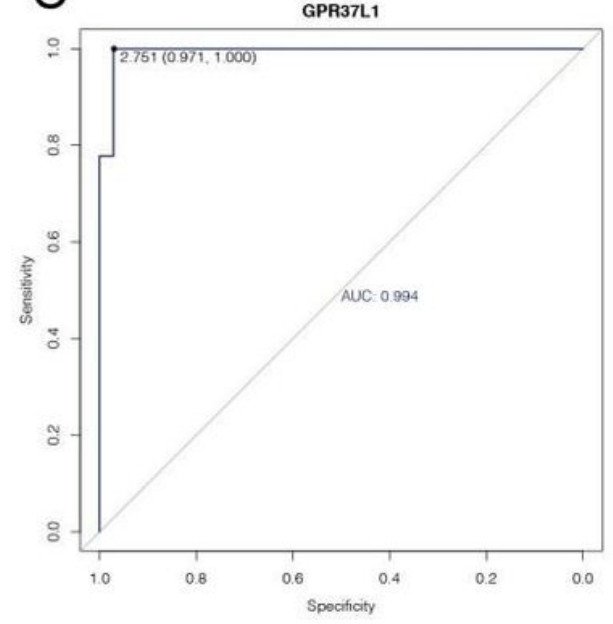

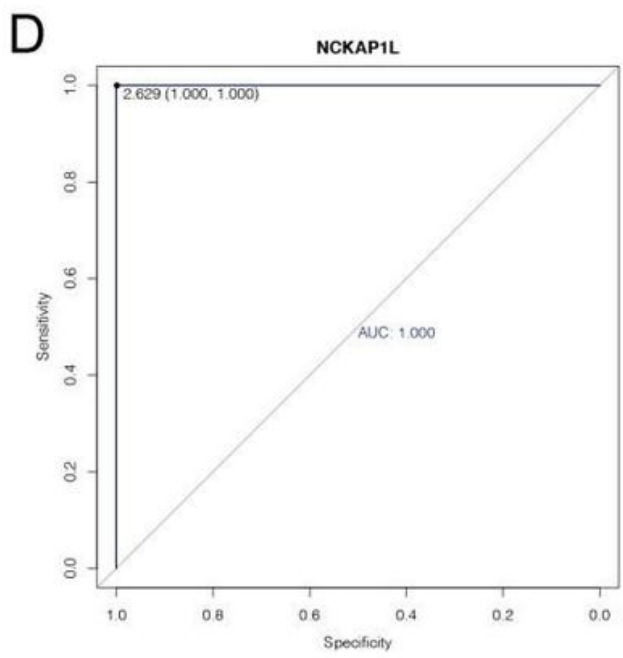

$\mathrm{E}$

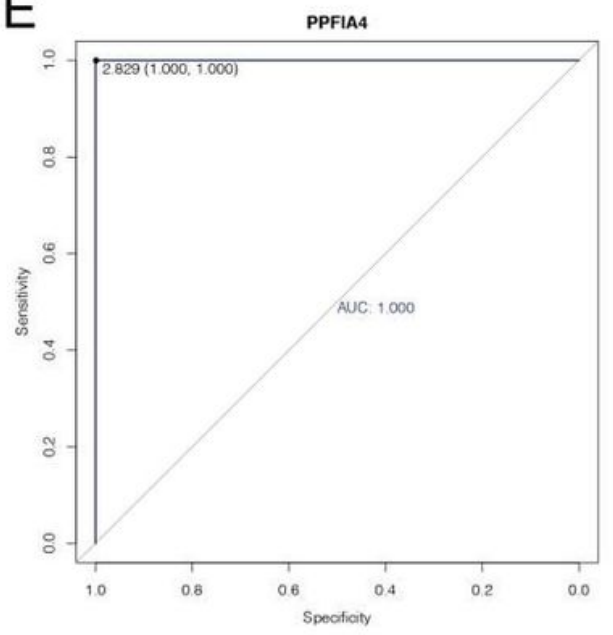

\section{Figure 9}

Diagnostic capacity of hub genes. A-E: ROC analysis to evaluate the diagnostic value of 5 hub genes. The AUC of GPR37L1 was 0.994 , and the AUC of other genes was 1.000 .

\section{Figure 10}

Immune infiltration analysis. A: The ImmuneScore in the two groups with different expressions of NCKAP1L. B: The StromalScore in the two groups with different expressions of NCKAP1L. C: The ESTIMATEScore in the two groups with different expressions of NCKAP1L. D: The difference in abundance of 28 immune-related gene sets in normal and PA tumor samples analyzed by ssGSEA. E: The lollipop plot for the correlation between NCKAP1L and immune cells ratio.

\section{Supplementary Files}

This is a list of supplementary files associated with this preprint. Click to download.

- SupplementaryFig.rar

- SupplementaryTable.rar 